\title{
Formation of particulate microporous poly(vinylidene fluoride) membranes by isothermal immersion precipitation from the 1-octanol/ dimethylformamide/poly(vinylidene fluoride) system
}

\author{
Liao-Ping Cheng ${ }^{\mathrm{a}, *}$, Tai-Horng Young ${ }^{\mathrm{b}}$, Lin Fang ${ }^{\mathrm{a}}$, Jy-Jie Gau $^{\mathrm{a}}$ \\ ${ }^{a}$ Department of Chemical Engineering, Tamkang University, Taipei Hsien, Taiwan \\ ${ }^{\mathrm{b}}$ Center for Biomedical Engineering, College of Medicine, National Taiwan University, Taipei, Taiwan
}

Received 11 July 1997; revised 16 February 1998; accepted 2 June 1998

\begin{abstract}
Phase diagrams were determined for poly(vinylidene fluoride) (PVDF) and a terpolymer of vinylidene fluoride, hexafluoropropylene, and tetrafluoroethylene (VDF/HFP/TFE) in solutions composed of 1-octanol and dimethylformamide at $25^{\circ} \mathrm{C}$. From the measured gelation and liquid-liquid phase separation data, binary interaction parameters were computed using a modified Flory-Huggins theory. PVDF microporous membranes were then prepared by isothermal immersion precipitation processes in various doping conditions. The formed membrane exhibited a particulate morphology characterized by a uniform package of spherical particles of identical size. These particles were identified to be full spherulites of PVDF using scanning electron microscopy, differential scanning calorimetry and small angle light scattering techniques. (C) 1999 Elsevier Science Ltd. All rights reserved.
\end{abstract}

Keywords: Poly(vinylidene fluoride); Phase diagram; Membrane

\section{Introduction}

Poly(vinylidene fluoride) (PVDF) is an acid-resistant, inert, semicrystalline polymer [1] that is also extensively used as a membrane material in biomedical applications $[2,3]$. There have been commercial ultrafiltration and microfiltration membranes manufactured by the Millipore Corporation using the immersion precipitation method based upon the patents of Grandine [4,5]. Munari and co-workers addressed the formation of both PVDF and sulfonated PVDF membranes and considered the membranes prepared from water/(TEP, DMF, DMSO)/PVDF systems as the 'state of the art' ultrafiltration membranes [6-8]. Recently, Jian and Pintauro [9] used PVDF membranes in pervaporation processes to extract minuscule amounts of benzene dissolved in water.

In spite of the technological importance of PVDF membranes, the subject concerning the mechanism of PVDF membrane formation is rarely properly discussed in the literature; particularly, the role crystallization plays during the precipitation process. In 1995, Soh et al. [10] first

\footnotetext{
* Corresponding author. Tel.: +886-2-6215656; Fax: +886-2-6209887; E-mail: Ipcheng@sigma.che.tku.edu.tw.
}

presented the phase diagram of the 1-octanol/dimethylformamide/PVDF system. In their publication, both the liquidliquid phase separation and the crystallization boundaries were reported over a wide temperature range. However, discrepancies have appeared between their experimental gelation data and computed crystallization lines. Also, the relation between ternary phase behavior and membrane morphology was not discussed [10].

In the current work, an improved approach was taken to investigate the equilibrium phase behavior of the 1-octanol/ DMF/PVDF system. The results were then used to study the PVDF membrane formation mechanism and ultimately to optimize the membrane structure for microfiltration applications. A crystalline PVDF and a structurally similar but largely amorphous terpolymer of vinylidene fluoride (VDF), hexafluoropropylene (HFP) and tetrafluoroethylene (TFE) were employed to measure, respectively, the gelation and binodal phase separation boundaries. Computations of the amorphous phase behavior of PVDF were carried out based upon Flory-Huggins interaction parameters. Membranes were prepared in various doping conditions by the isothermal immersion precipitation method. An interesting membrane structure was discovered for the PVDF: the membrane looked like a packed bed consisting of spherical particles of 


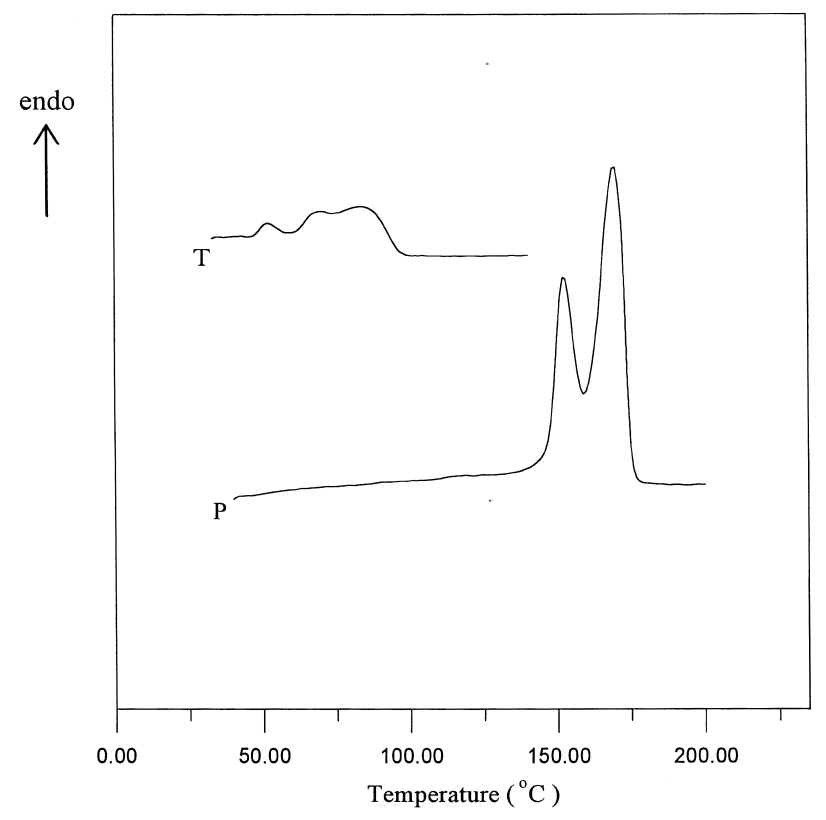

Fig. 1. DSC thermograms of PVDF and terpolymer. Curve P: PVDF (Kynar 961), $\Delta H=62.5 \mathrm{~J} / \mathrm{g}$. Curve T: terpolymer (Kynar 9301).

nearly identical size, somewhat similar to the particulate EVAL membranes reported previously [11]. The diameter of the particle can vary from ca. 0.3 to $2.5 \mu \mathrm{m}$, depending upon the preparation conditions. Using differential scanning calorimetry (DSC), scanning electron microscopy (SEM) and small angle light scattering (SALS) techniques, these particles were identified to be full spherulites of crystalline PVDF.

\section{Experimental}

\subsection{Materials}

PVDF (Kynar 961, intrinsic viscosity $=0.982 \mathrm{dl} / \mathrm{g}, M_{\mathrm{n}}=$ $298000 \mathrm{~g} / \mathrm{mol}$ ) was obtained from Pennwalt Corp. Terpolymer of VDF, HFP and TFE (Kynar 9301, VDF/ $\mathrm{HFP} / \mathrm{TFE}=60: 20: 20$, intrinsic viscosity $=0.4 \mathrm{dl} / \mathrm{g}, M_{\mathrm{n}}=$ $79200 \mathrm{~g} / \mathrm{mol}$ ) was supplied free of charge by Elf Atochem Inc. Kynar 961 (called PVDF hereafter) is crystalline, whereas Kynar 9301 (called terpolymer hereafter) is largely amorphous, as is evident from the DSC thermograms of these polymers, shown in Fig. 1. N,N-Dimethylformamide (DMF; Baker Analyzed, reagent grade, $d=0.944 \mathrm{~g} / \mathrm{ml}$ ) and 1-octanol (Reidel-de Haen, reagent grade, $d=0.82 \mathrm{~g} / \mathrm{ml}$ ) were used as solvent and nonsolvent for the polymers, respectively. All materials were used as received.

\subsection{Gelation boundary}

The gelation boundary at $25^{\circ} \mathrm{C}$ of PVDF in 1-octanol/
DMF solutions were determined by the so-called "cloud point' method [12]. A specific amount of PVDF (dried in a vacuum oven at $70^{\circ} \mathrm{C}$ ) was mixed with $\mathrm{DMF}$ and sealed in a Teflon-lined bottle $(20 \mathrm{ml})$. This mixture was then blended at elevated temperature until the polymer was completely dissolved. To this solution was added a known quantity of nonsolvent. Local precipitation may be observed. The mixture was agitated in a roll mill (at ca. $65-100^{\circ} \mathrm{C}$ ) until a clear homogeneous solution was obtained. This solution was then put in a thermostatically controlled oven maintained at $25^{\circ} \mathrm{C}$ for a period of 7 days. The equilibrium gelation points were identified as the compositions at which homogeneous solutions just began to precipitate into a gel.

\subsection{Liquid-liquid phase separation boundary (binodal)}

The binodal was measured only for the amorphous terpolymer system. Because phase-separated solutions cannot be easily mechanically separated as the polymer content exceeds ca. $40 \mathrm{wt} \%$, two methods were employed to obtain the binodal: (i) the cloud point method at low polymer contents; (ii) the equilibrium sorption method at high polymer contents [12].

1. Cloud point method: a series of ternary solutions were prepared and then put in a thermostat at $25^{\circ} \mathrm{C}$, as in the aforementioned procedure. Unstable solutions underwent phase separation into two clear liquid layers. The binodal was defined as the compositions of the phase separation points at $25^{\circ} \mathrm{C}$.

2. Equilibrium absorption method: appropriate amounts of PVDF, 1-octanol and DMF were sealed in a glass bottle, and were roll mixed for 3 days at $65^{\circ} \mathrm{C}$ and then put in a thermostat at $25^{\circ} \mathrm{C}$ for another 7-10 days. The swollen polymer gel was mechanically separated by centrifugation from the dilute phase, which contained essentially only 1-octanol and DMF. The composition of the dilute phase was determined by gas chromatography and the mass fraction of polymer in the swollen polymer gel was determined by drying to a constant weight. Knowing the overall composition, the tie line compositions were computed for each overall composition.

\subsection{Characterization of PVDF gels by DSC and SALS}

The crystalline characters of PVDF gels were analyzed using both DSC (DSC 200, Netzsch, Germany) and SALS methods [13]. DSC measurements were carried out in a sealed sample pan at a scanning rate of $10^{\circ} \mathrm{C} / \mathrm{min}$ over the temperature range of $10-100^{\circ} \mathrm{C}$. For the SALS imaging, a small amount of gel sample (the gel is transparent) was pressed tightly between two glass plates and then placed between two crossed polarizers. An $\mathrm{He}-\mathrm{Ne}$ laser was 


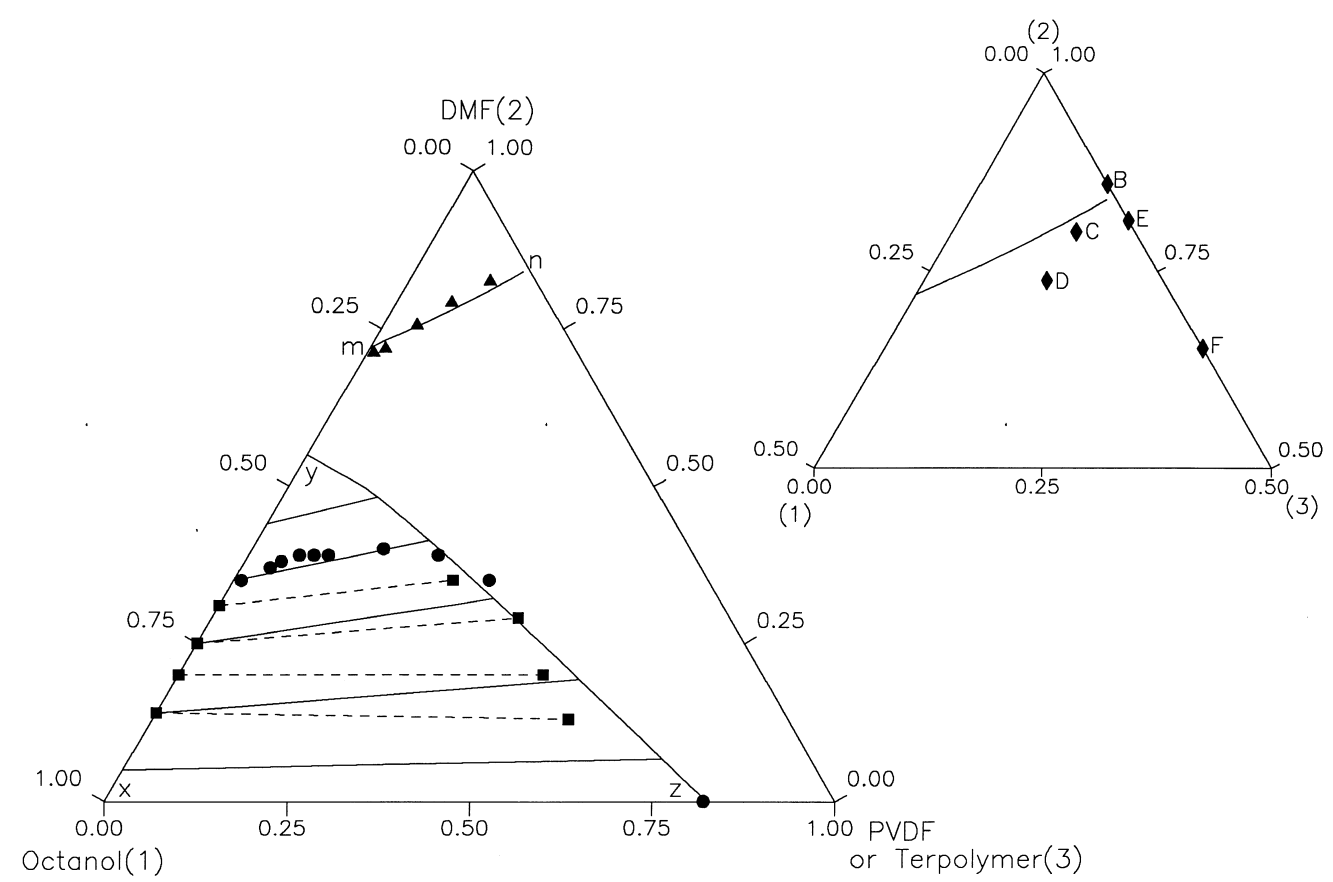

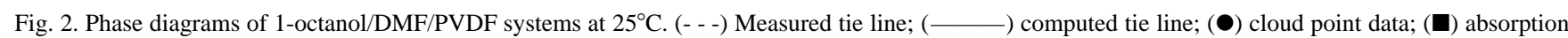
equilibrium data; $(\boldsymbol{\Delta})$ measured gel points; $(\diamond)$ dope solutions for membrane preperation. Line 'mn': computed crystallization line. Line 'xyz': computed binodal.

used as the light source and the scattering image formed on a ground glass was photographed using a macro lens [13].

\subsection{Membrane preparation and characterization}

Membranes were prepared using the direct immersion precipitation method at $25^{\circ} \mathrm{C}$. A homogeneous dope composed of polymer (Kynar 961 or Kynar 9301), DMF and 1-octanol was spread uniformly on a glass plate to form a laminate (ca. $250 \mu \mathrm{m}$ ), which was then immersed immediately into a 1-octanol bath to effect polymer precipitation. Compositions of the dopes are shown as filled diamond symbols $(\checkmark)$ in the phase diagram of Fig. 2 (upper right corner). Dope B for PVDF and dope F for terpolymer represent homogeneous polymer solutions in a good solvent. Dopes C, D and E for PVDF are solutions that are in a state of 'incipient precipitation' with respect to the crystallization of PVDF. These dopes are commonly observed to precipitate upon standing (overnight) and they have to be heated to ensure complete dissolution before immersing at $25^{\circ} \mathrm{C}$ into the octanol bath. The membrane formed was soaked in ethanol to remove 1-octanol and residual DMF, and then in acetone and dried in the open air. SEM photomicrographs of the dried membranes were taken in different views. Melting temperature and enthalpy of fusion of the membranes were determined by DSC at the rate of $10^{\circ} \mathrm{C} / \mathrm{min}$. Water permeation rates of the membranes were measured with a dead-end type standard Amicon cell (effective area $=11.9 \mathrm{~cm}^{2}$ ) at different filtration pressures.

\section{Results and discussion}

3.1. Phase boundaries of PVDF and terpolymer in octanol/ DMF at $25^{\circ} \mathrm{C}$

In Fig. 2, phase diagrams at $25^{\circ} \mathrm{C}$ are shown for both crystalline PVDF and amorphous terpolymer in 1-octanol/ DMF systems. The experimental liquid-liquid phase

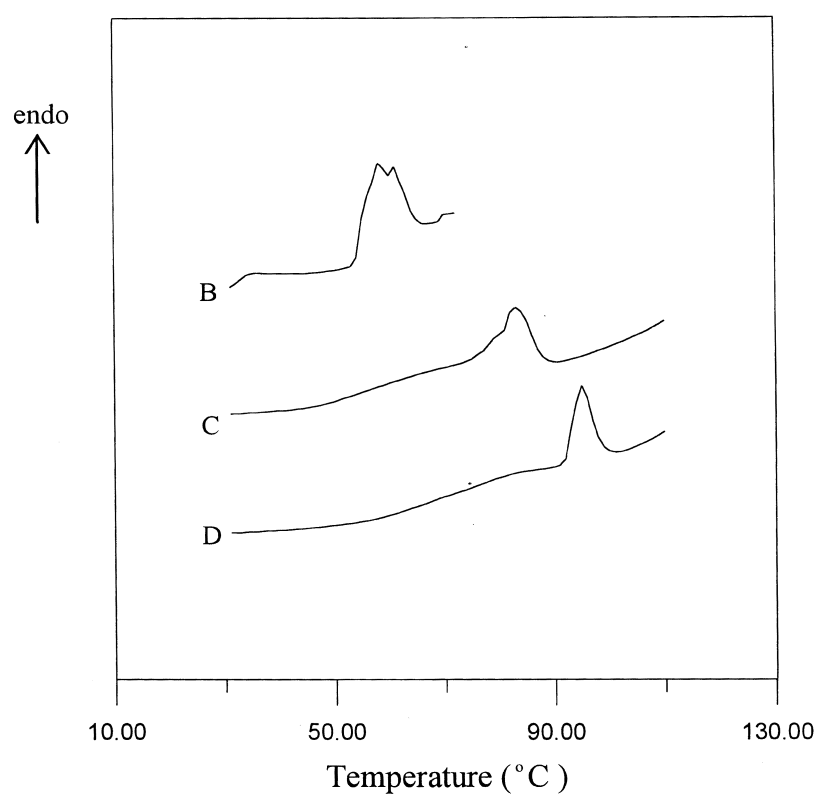

Fig. 3. DSC thermograms of crystalline gels. Gel B: 5 wt $\%$ octanol, 71 wt $\%$ DMF, $24 \mathrm{wt} \%$ PVDF. Gel C: $15 \mathrm{wt} \%$ octanol, $64 \mathrm{wt} \%$ DMF, $21 \mathrm{wt} \%$ PVDF. Gel D: 20 wt $\%$ octanol, 60 wt $\%$ DMF, 20 wt $\%$ PVDF. 


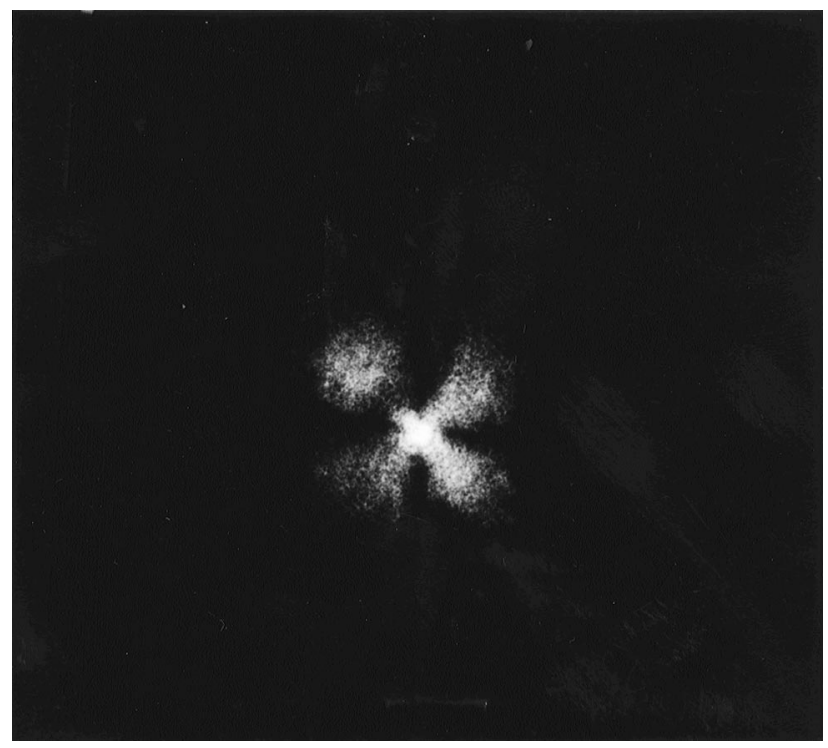

Fig. 4. The experimental SALS image of a PVDF gel (point D in Fig. 2)

separation data points represent the compositions of the two equilibrium liquid phases measured for the terpolymer. The equilibrium data points are joined by tie lines and the collection of all tie line ends forms the binodal. For crystalline PVDF in 1-octanol/DMF, solid-liquid equilibrium is established. The filled triangle data points symbolize the compositions of the liquid phase which coexist in equilibrium with pure PVDF. Since this PVDF disperses uniformly in the liquid phase, the specimen appears in the form of a gel. The crystalline character of the gels has been examined by DSC. In Fig. 3, DSC thermograms are shown for several of the equilibrium gels formed at $25^{\circ} \mathrm{C}$. The endotherms show that the melting temperatures are higher for those gels containing more octanol (i.e. gels at a higher supersaturation with respect to crystallization of PVDF). This is consistent with the observations reported by Witte et al. [14], who used DSC to study the crystallization equilibrium of the poly(lactide)/chloroform/methanol system. The SALS image of a PVDF gel (point D in Fig. 2) is shown in Fig. 4. The four-clove pattern clearly indicates that this gel is composed of spherulites dispersed uniformly in the 1-octanol/DMF solution. As a dope solution made from this gel is immersed into a nonsolvent bath, the introduction of 1-octanol into the polymer solution causes a rapid crystallization of PVDF to form the particulate structure shown later in Fig. 9.

Table 1

Summary of interaction parameter data

\begin{tabular}{ll}
\hline Binary system & Interaction parameter \\
\hline 1-Octanol (1)/DMF (2) & $(0.64-0.47) /\left(1+5.42 h_{2}\right)$ \\
1-Octanol (1)/PVDF (3) & 1.35 \\
DMF (2)/PVDF (3) & $-1+0.5 \phi_{3}$ \\
\hline
\end{tabular}

$\phi_{i}$ : volume fraction of component $i(i=1$ : octanol; $i=2$ : DMF; $i=3$ : PVDF $) ; h_{2}=2 /\left(\phi_{1}+\phi_{2}\right)$.
Following a computational scheme developed previously $[11,12]$, the experimental gelation data were used to obtain the best fitted crystallization isotherm. Line 'mn' in Fig. 2 appears to match closely the experimental data points, suggesting that a reliable curve fitting has been obtained. The interaction parameter between polymer and solvent, $\chi_{23}$, was determined and the results are given in Table 1 . The concentration-dependent interaction parameter for the 1 -octanol/DMF binary pair, $\chi_{12}$, was obtained using vapor liquid equilibrium data generated by a computer code based on the UNIFAC method [15]. At $25^{\circ} \mathrm{C}$, the equilibrium absorption of 1-octanol by PVDF film was measured and used to calculate the interaction parameter, $\chi_{13}$ [11]. Other physical constants for computations are given in Table 2 . The heat of fusion of PVDF at the melting temperature, $\Delta H$, and the heat capacities in the solid and melt states were determined using DSC.

Using the interaction parameters shown in Table 1, the theoretical liquid-liquid phase equilibrium boundary of crystalline PVDF in 1-octanol/DMF was determined by solving chemical potential equations modified from Flory-Huggins theory (Eqs. (2)-(4) in Ref. [12]). The calculated binodal (line 'xyz') and tie lines (solid lines) are shown in Fig. 2. It can be observed that line 'xyz' agrees reasonably well with the measured binodal data points for terpolymer over a wide concentration range. This implies that the amorphous phase behavior of PVDF in 1-octanol/DMF solutions approaches that of the terpolymer. Since these two polymers have similar chemical structure, it is not surprising that their amorphous phase behaviors are close. Such a phenomenon has also been reported by Cheng et al. [12] for the water/formic acid/aliphatic polyamide systems. However, contrary to our present result, Witte pointed out recently that the liquid-liquid phase equilibrium boundaries of crystalline poly(L-lactide) and amorphous poly(DL-lactide) deviated significantly from each other, even though these polymers differ only in the spatial arrangements of atoms [16]. In the region near the intercept point of the binodal in Fig. 2, the measured data of the terpolymer are lower than those of the calculated results for PVDF. This is mainly due to the fact that the molecular weight of PVDF is much higher than that of the terpolymer. This has been addressed by Koningsveld [17], where molecular weight affects the amorphous phase behavior of nonsolvent/solvent/polymer systems most significantly in

Table 2

Physical constants for PVDF polymer

\begin{tabular}{lllll}
\hline$T_{\mathrm{m}}$ & $\Delta H$ & $A$ & $B$ & $V_{\mathrm{u}}$ \\
\hline 170 & 62.5 & 135 & -0.38 & 36.178 \\
\hline
\end{tabular}

$T_{\mathrm{m}}\left({ }^{\circ} \mathrm{C}\right)$ : melting temperature; $\Delta H(\mathrm{~J} / \mathrm{g})$ : enthalpy of fusion; $C_{\mathrm{c}}\left(\mathrm{J} / \mathrm{mol}{ }^{\circ} \mathrm{C}\right)[a$ $+b T]$ heat capacities in the solid states; $C_{\mathrm{m}}\left(\mathrm{J} / \mathrm{mol}{ }^{\circ} \mathrm{C}\right)[c+d T]$ : heat capacities in the melt states; $A=c-a, B=d-b ; V_{\mathrm{u}}$ : molar volume per polymer repeat unit. 

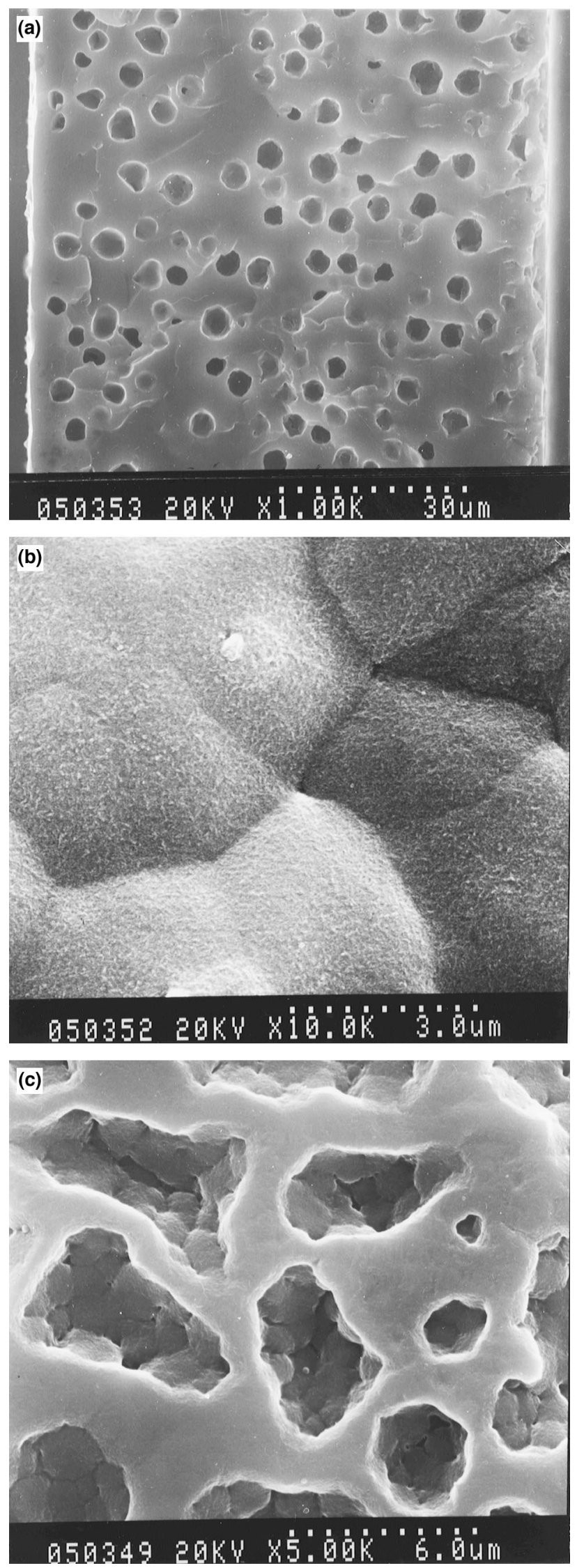

the region near the intercept point. As the molecular weight of the polymer is increased, the intercept point shifts upwards.

\subsection{Morphology of amorphous terpolymer membranes}

In Fig. 5(a), the cross-section of the membrane formed by immersing a $50 \mathrm{wt} \%$ terpolymer solution (point F in Fig. 2) into a pure 1-octanol bath is shown. This cross-section shows a porous structure consisting of cellular pores enclosed in a continuous polymer matrix. Since the terpolymer has a low level of crystallinity (Fig. 1 shows a slight DSC melting endotherm for the terpolymer), it follows that the process of liquid-liquid phase separation dominates the precipitation process. Unlike ordinary amorphous membranes, the pores of this membrane are rather circular and all of them are isolated. The pore density of the membrane is very low, which suggests that liquid micelles are not extensively formed during the nucleation stage of membrane formation. Fig. 5(b) and (c) represent the top and bottom surfaces of this membrane. The top surface is essentially nonporous. However, because of the residual crystallinity in the terpolymer, this surface exhibits a polygonal plate structure which resembles the surface of an aliphatic polyamide membrane formed by nucleation and growth of two-dimensional spherulites [18]. The bottom surface is porous and composed of cellular pores originating from the liquid-liquid phase separation. The walls surrounding the cells are particulate and are formed from crystallization after the liquid-liquid phase separation is completed [18].

\subsection{Morphology of crystalline PVDF membranes}

In contrast to the cellular morphology demonstrated above for the amorphous terpolymer, crystalline PVDF membranes are characterized by the so-called 'particulate' morphology. PVDF membranes were prepared by immersing various PVDF dopes into a 1-octanol bath. The microscopic structures of the formed membranes are shown in Figs 6, 8 and 9. The case of employing a $23 \mathrm{wt} \%$ PVDF dope (point B in Fig. 2) is shown in Fig. 6. This membrane is skinless and is very uniform in all dimensions. The building units are spherical particles of approximately equal size (ca. $0.8 \mu \mathrm{m}$ diameter). This suggests that all of the particles are grown from individual PVDF nuclei created roughly at the same time. These nuclei then grow radially until their fronts meet and join with adjacent particles. In Fig. 6, cellular pores (an imprint of the occurrence of liquid-liquid phase separation) are not evident. This affirms the domination of crystallization during the precipitation process. The DSC melting endotherms of various membranes (the morphology of these membranes will be discussed later)

Fig. 5. SEM photomicrographs of the membrane formed by immersing a $50 \mathrm{wt} \%$ terpolymer solution (point $\mathrm{F}$ in Fig. 2) into a 1-octanol bath. (a) Cross-section. (b) Top surface. (c) Bottom surface. 

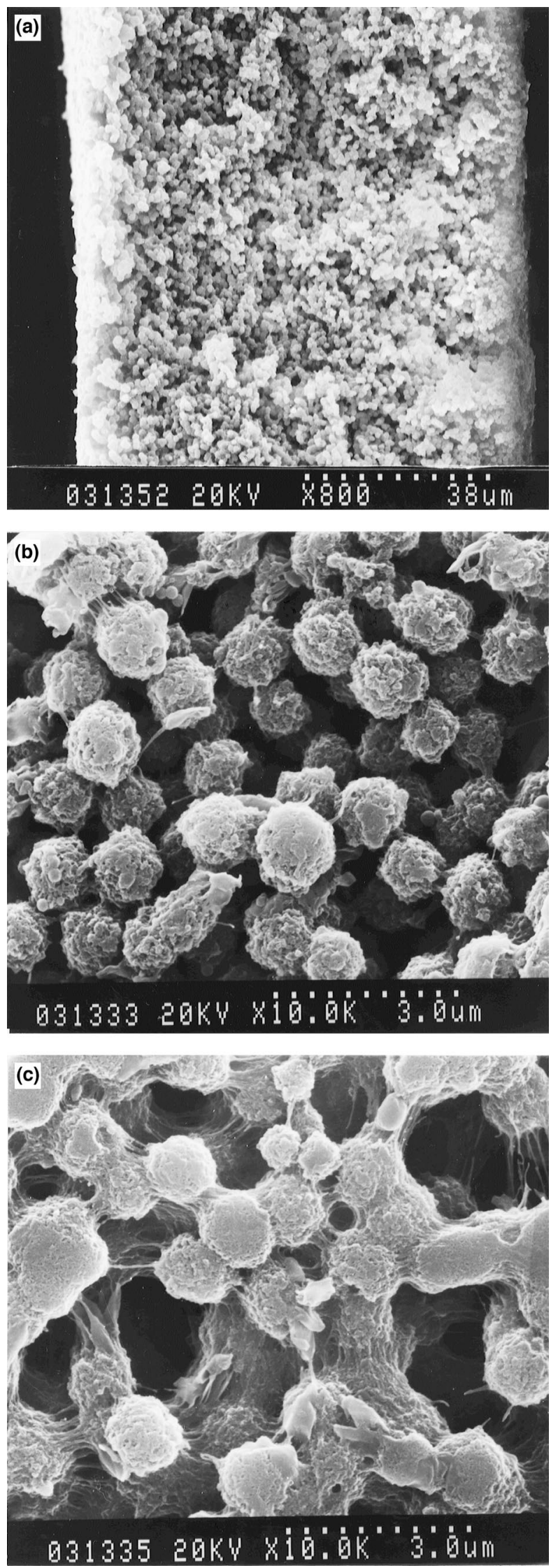

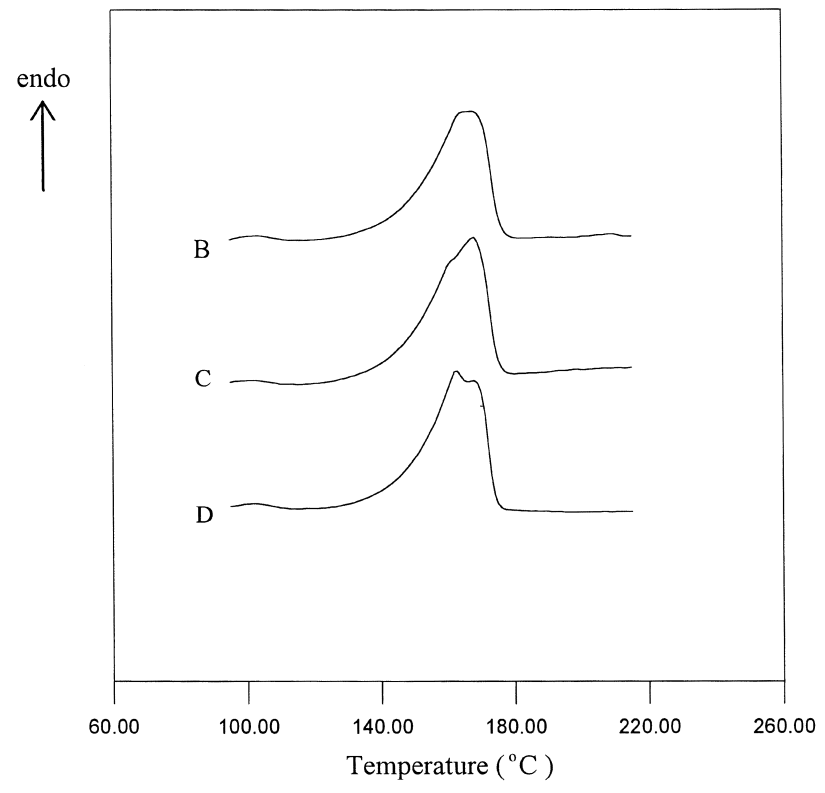

Fig. 7. DSC thermograms of membranes prepared from various dopes. Curves B, C and D represent dopes B, C and D in Fig. 2, respectively. $\Delta H \cong 61 \mathrm{~J} / \mathrm{g}$ and $T_{\mathrm{m}}=170^{\circ} \mathrm{C}$ for all membranes, respectively.

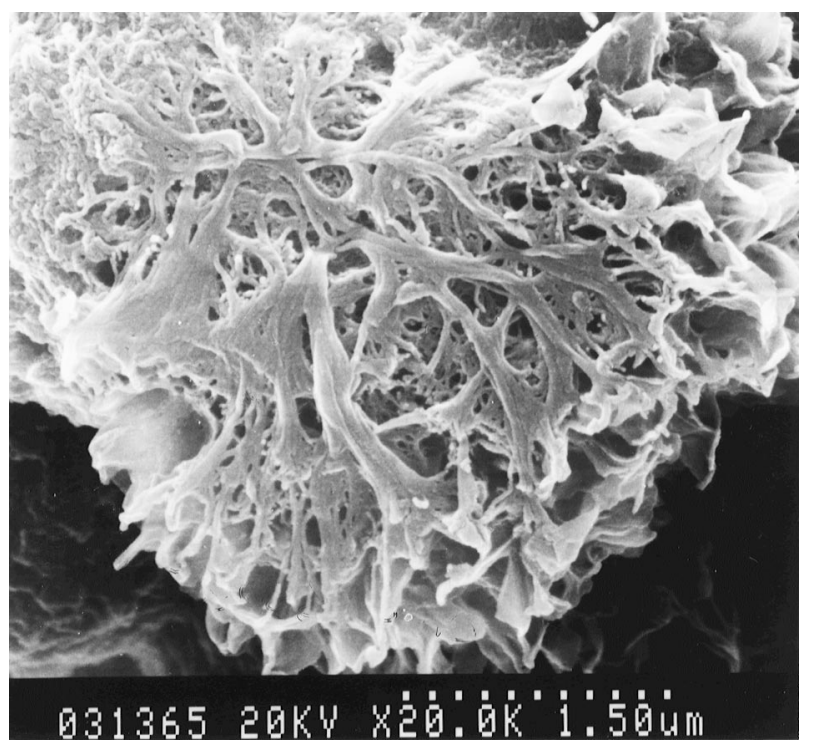

Fig. 8. SEM photomicrograph of a PVDF crystallite. Dope: point E in Fig. 2.

are shown in Fig. 7. It appears that all membranes have an enthalpy of fusion similar to that of the original PVDF pellet (Fig. 1). However, the first melting peak of the PVDF pellet at $153^{\circ} \mathrm{C}$ is absent from the endotherms of the membranes. This implies that, during membrane formation, crystallinity of the PVDF is maintained but the crystal form has changed. In Fig. 8, an SEM photomicrograph of a

Fig. 6. SEM photomicrographs of the membrane formed by immersing a $23 \mathrm{wt} \%$ PVDF dope (point B in Fig. 2) into a 1-octanol bath. (a) Crosssection. (b) Top surface. (c) Bottom surface. 

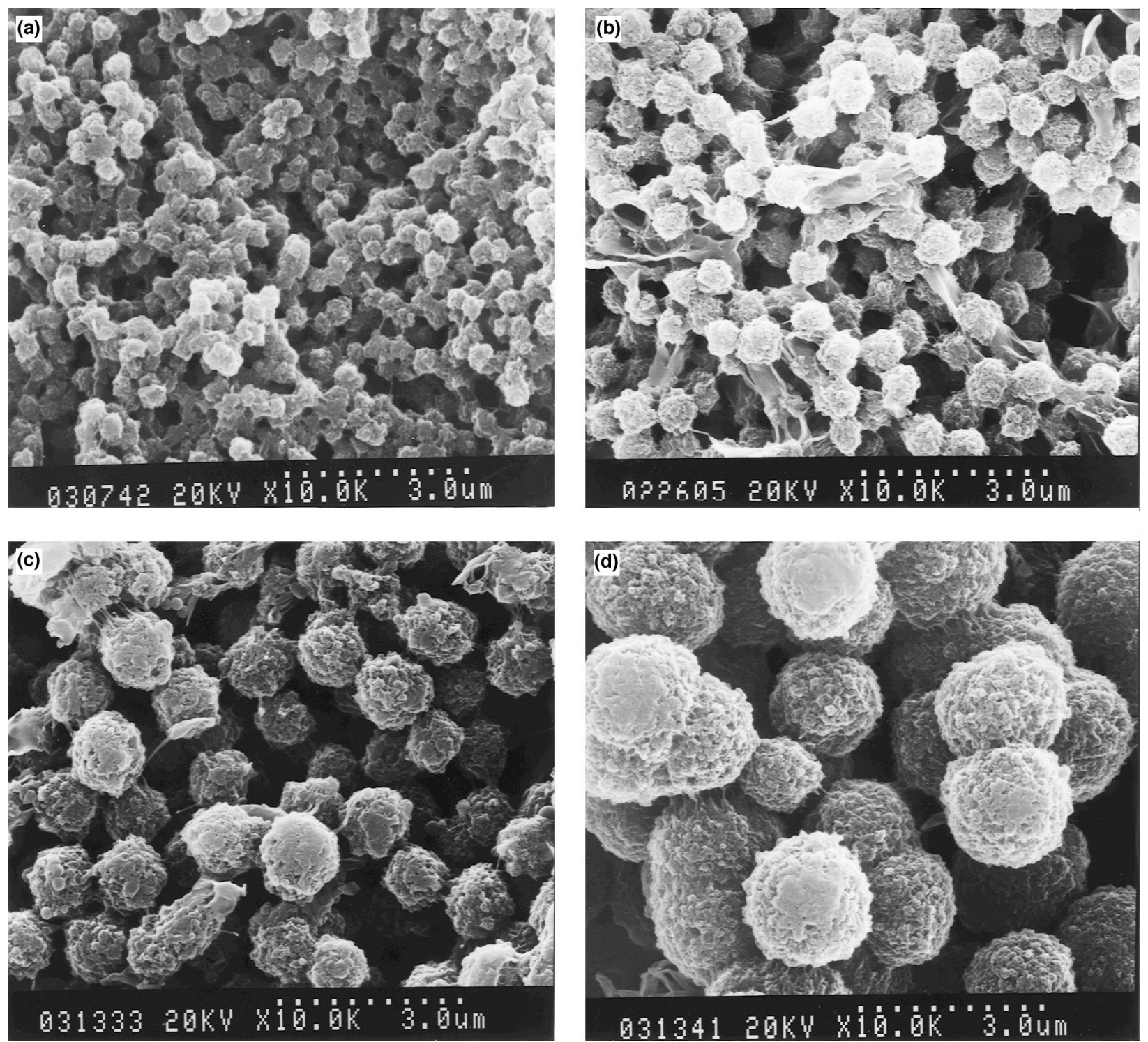

Fig. 9. SEM photomicrographs of membranes formed by immersing various dopes into a 1-octanol bath. (a) PVDF: 23 wt\%; 1-octanol: 10 wt\%; point D in Fig. 2. (b) PVDF: 23 wt \%; 1-octanol: 5 wt $\%$; point C in Fig. 2. (c) PVDF: 23 wt $\%$; 1-octanol: 0 wt $\%$; point B in Fig. 2. (d) PVDF: 30 wt $\%$; octanol: 0 wt $\%$; point $\mathrm{E}$ in Fig. 2 .

PVDF crystallite is shown, which demonstrates unambiguously the dendritic character of this crystallite $[19,20]$. As a PVDF nucleus grows in the immersion precipitation process, it tends to follow the nature of a spherulite from rod to sheaf, finally becoming a complete spherulite [18-20].

The mechanical strength of a microporous membrane depends heavily upon the links between polymer particles. For the present PVDF membranes, the linkage points are relatively limited only on the interface between spherical particles. As a result, their strength is not as satisfactory as that of nylon microporous membranes, whose rod-like particles intertwine considerably [18,21-23]. However, the strength of PVDF membranes can be increased without significantly changing the microscopic structure simply by a post-heat treatment process at $165^{\circ} \mathrm{C}$. As will be shown later, heat-treated membranes are strong enough to endure microfiltration operations.

\subsection{Size of particles in the crystalline membranes}

For a crystalline polymer, precipitation may take place by means of liquid-liquid phase separation, crystallization or certain combinations of both to yield sophisticated morphologies $[18,21,22]$. As shown above, PVDF membranes with a uniform microporous morphology were found to be formed in a manner dictated by the crystallization process. By varying the compositions of the dopes, membranes comprised of spherulitic particles over a wide range of sizes (ca. $0.3-2.5 \mu \mathrm{m}$ diameter) were prepared. Fig. 9(a)-(c) represent the cases of immersing $23 \mathrm{wt} \%$ PVDF dopes 


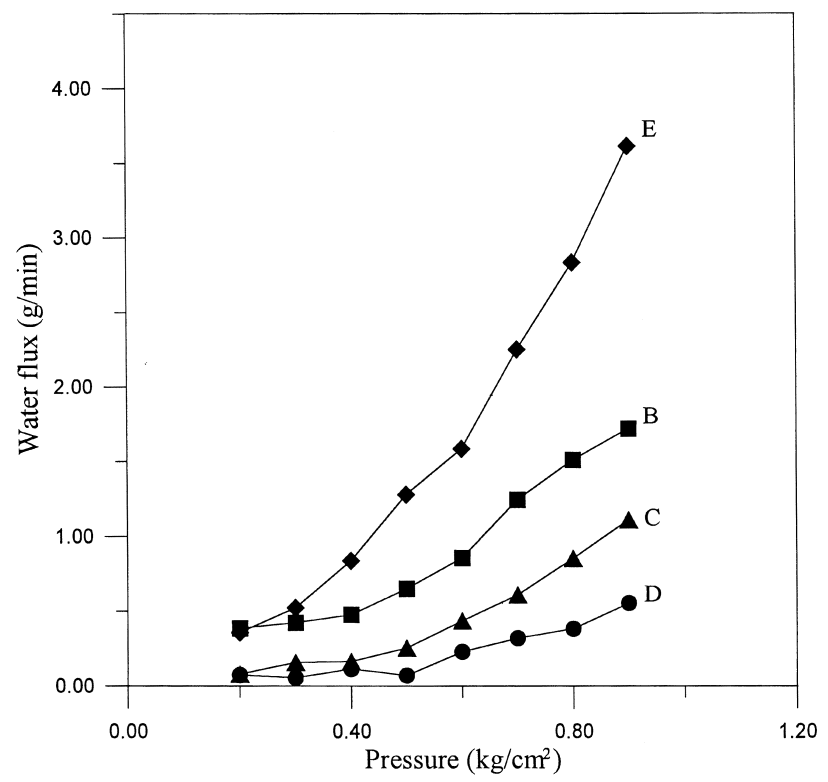

Fig. 10. The experimental water flux data. Lines B, C, D and E represent membranes prepared from dopes B, C, D and E in Fig. 2, respectively.

containing different amounts of 1-octanol (points B, C and D in Fig. 2) into a 1-octanol bath. Fig. 9(c) has been shown previously in Fig. 6(b) and is shown again here for comparison. Dopes $\mathrm{C}$ and $\mathrm{D}$ were prepared such that they were in a state of 'incipient precipitation' with respect to crystallization of PVDF. These dope solutions will gel upon standing, which suggests that the polymer is in a poor dissolution state and there might exist a certain degree of polymer aggregation in these dope solutions. Fig. 9(a)-(c) indicate that the size of the particles decreases (or the number of particles increases) as the amount of 1-octanol in the dope is increased. In other words, the nucleation density, which depends upon the degree of supersaturation with respect to crystallization, is lower for dopes containing less 1-octanol. For an isothermal system, such as the current case, the degree of supersaturation of a dope is measured as the distance below the crystallization boundary. The present results appear to be consistent with the cases of thermalinduced crystallization processes (e.g., shock freezing) in which the amount of nuclei is increased (or the size of crystallites is decreased) as the degree of supercooling is increased [20,24,25].

As the PVDF content in the dope is increased from 20 to $30 \mathrm{wt} \%$ (point E in Fig. 2), the spherical particles become very large (ca. $2.1 \mu \mathrm{m}$ diameter), as shown in Fig. 9(d). Since dope $\mathrm{E}$ is at a higher supersaturation with respect to crystallization than dope A (see Fig. 2), this result seems to contradict the common observation in the thermal crystallization processes mentioned above. In our opinion, the viscosity of the dope plays a rather important role in the present situation. As a subcritical 'embryo' is formed from a concentration fluctuation, it may grow into a nucleus or redissolve into the liquid, depending on whether the polymer molecules can successfully deposit and then rearrange themselves on the 'embryo'. In a very viscous medium, such as the $30 \mathrm{wt} \%$ polymer solution, translation of PVDF molecules by diffusion is not as effective as in the lower concentration cases. As a result, the rate of formation of nuclei is reduced even with favorable thermodynamic conditions.

For those particulate membranes shown above, the effective pore size changes in accordance with the size of the spherical particle. Water fluxes of various heat-treated membranes (formed from dopes B, C, D and E) were measured in a standard Amicon cell for the pressure range of $0.2-0.9 \mathrm{kgw} / \mathrm{cm}^{2}$. The results are shown in Fig. 10. As is evident from this figure, the water fluxes of the tested membranes follows the order: $\mathrm{E}>\mathrm{B}>\mathrm{C}>\mathrm{D}$. This appears to agree with the morphology of these membranes as shown in Fig. 9, wherein the diameter of the particle (or equilivantly the pore size) also decreases in the same order.

\section{Conclusion}

In this work, equilibrium phase boundaries were determined both for crystalline PVDF (Kynar 961) and amorphous terpolymer (Kynar 9301) in 1-octanol/DMF solutions at $25^{\circ} \mathrm{C}$. The normally inaccessible amorphous phase boundary of the PVDF system was calculated by interaction parameters fitted to the crystallization data. Thus, the obtained binodal and tie lines were found to agree with those of the terpolymer system. Membranes were prepared by the direct immersion precipitation method. The terpolymer membrane exhibited an asymmetric morphology composed of a skin and a cellular sublayer, whereas the PVDF membranes are skinless and uniform, being composed of equal-sized spherical particles that bind together to form a bi-continuous structure as in a microporous membrane. DSC, SEM and SALS studies indicate that these particles are actually full spherulites originating from crystallization during the precipitation process. The particle size was found to undergo a significant change by altering the nonsolvent and polymer concentrations in the dopes. For dopes that contain less 1-octanol or having a higher PVDF concentration, particle sizes and therefore pore diameters are found to be larger.

\section{Acknowledgements}

We thank the National Science Council of Taiwan, Republic of China, for financial support of this research (NSC 86-2216-E-032-002).

\section{References}

[1] Lovinger AJ. In: Bassett DC, editor. Development in crystalline polymers, vol. 1. London: Applied Science, 1982. 
[2] Guenard V, Valentini RF, Aebischer P Biomaterials 1991;12:259.

[3] Chen H, Soldani G, Galletti PM, Goddard M ASAIO J 1992; 38:201.

[4] Grandine JD. United States Patent 4203847, 1977.

[5] Grandine JD. United States Patent 4203848, 1977.

[6] Munari S, Bottino A, Camera Roda G, Capannelli G Desalination 1990;77:85.

[7] Munari S, Bottino A, Capannelli G J Membr Sci 1983;16:181.

[8] Bottino A, Camera Roda G, Capannelli G, Munari S J Membr Sci 1991;57:1.

[9] Jian K, Pintauro PN J Membr Sci 1993;85:301.

[10] Soh YS, Kim JH, Gryte CC Polymer 1995;36:3711.

[11] Young TH, Lai JY, You WM, Cheng LP Equilibrium phase behavior of the membrane forming water-DMSO-EVAL copolymer system. J Membr Sci 1997;128:55.

[12] Cheng LP, Dwan AW, Gryte CC J Polym Sci, Part B: Polym Phys 1994;32:1183.

[13] Meeten GH. Optical properties of polymers. London: Elsevier Applied Science, 1986.
[14] Witte PV, Boorsma A, Esselbrugge H, Dijkstra PJ, Berg JWA Macromolecules 1996;29:212.

[15] Sandler SI. Chemical and engineering thermodynamics. New York: Wiley, 1989.

[16] Witte PV, Esselbrugge H, Dijkstra PJ, Feijen J, Berg JWA J Polym Sci, Part B: Polym Phys 1996;34:2553.

[17] Koningsveld R Adv Colloid Interface Sci 1968;2:151.

[18] Cheng LP, Dwan AW, Gryte CC J Polym Sci, Part B: Polym Phys 1995;33:211.

[19] Woodward AE. Understanding polymer morphology. New York: Hanser, 1995.

[20] Wunderlich B. Macromolecular physics. New York: Academic Press, 1973.

[21] Bulte AM, Mulder MHV, Smolders CA Polymer 1996;37:1647.

[22] Knight RA. United States Patent 5084179, 1992.

[23] Pall DJ. United States Patent 4340479, 1982.

[24] Ueberreiter K. In: Crank J, editor. Diffusion in polymers, chap. 7. London: Academic Press, 1968.

[25] Zettlemoyer AC. Nucleation. New York: Marcel Dekker, 1969. 